\title{
Neurogenic motor evoked potentials: role in brachial plexus surgery
}

\author{
Case report
}

\author{
Lee M. Burkholder, B.Sc., David A. Houlden, Ph.D., \\ RAJIV MIDHA, M.D., M.Sc., F.R.C.S.(C), ERIN WEISS, M.Sc., \\ AND Marco VenNetTilli, B.Sc.
}

\author{
Division of Neurosurgery and Neuroscience Research Program, Sunnybrook and Women's College \\ Health Sciences Centre, University of Toronto, Ontario, Canada
}

\begin{abstract}
$\checkmark$ Peripheral nerve graft repair after severe brachial plexus injury is futile if there is degeneration of motor fibers in the proximal nerve stump to which the graft must be attached. Traditional intraoperative neurophysiological assessment methods like nerve action potential (NAP) and somatosensory evoked potential (SSEP) monitoring have been used to evaluate proximal nerve stump integrity, but these methods do not allow evaluation of the integrity of motor fibers back to the anterior horn cell. Consequently, the authors used transcranial electrical stimulation and recorded neurogenic motor evoked potentials (MEPs) directly from the brachial plexus in a patient undergoing surgical repair of a complete upper brachial plexus injury (Erb palsy) to assess the functional continuity of motor fibers. In addition, selected elements of the brachial plexus were directly stimulated, and NAPs were recorded. Finally, SSEPs were recorded from the scalp after stimulation of selected elements of the brachial plexus. Neurogenic MEPs were present from the medial cord of the brachial plexus, but not the middle or upper trunk; NAPs were present from the lateral and posterior cords after middle trunk stimulation, but absent after upper trunk stimulation; and SSEPs were present after medial cord stimulation but absent after stimulation of the upper and middle trunks. For the first time, neurogenic MEPs were coupled with NAPs and SSEPs to evaluate successfully the functional status of motor fibers back to the anterior horn cell for accurate localization of the lesion sites.
\end{abstract}

\section{KEY WoRDS • brachial plexus - root avulsion • motor evoked potential • nerve action potential - somatosensory evoked potential • transcranial electrical stimulation}

$\mathrm{I}$ $\mathrm{T}$ is imperative to evaluate accurately the type and level of lesions involved in brachial plexus injury, because management and prognosis of such an injury is determined by the distinction between proximal (preganglionic) and distal (postganglionic) lesion sites. ${ }^{1}$ For example, in cases of postganglionic lesions in which the nerve rootlets are continuous with the spinal cord, direct nerve repair with or without grafts to promote regeneration of degenerated nerve fibers is possible. In the case of preganglionic lesions, however, in which the nerve roots are avulsed from the spinal cord, indirect reconstructive strategies, including neurotization and tendon or muscle transfers, are necessary to restore motor function. Imaging technology does not allow us to evaluate the functional continuity of either the sensory or motor fibers, and it is not infallible for diagnosing nerve root avulsions, ${ }^{1}$ so intraoperative neurophysiological studies should be performed to confirm the preoperative diagnoses that are based on clinical and neuroimaging findings.

The neurophysiological methods commonly used intra-

Abbreviations used in this paper: $\mathrm{MEP}=$ motor evoked potential; $\mathrm{NAP}=$ nerve action potential; $\mathrm{SSEP}=$ somatosensory evoked potential; TCES = transcranial electrical stimulation. operatively are NAP and SSEP monitoring. The NAP is valuable for evaluating the functional status of mixed peripheral nerves and for localizing a lesion in the nerve branches distal to the dorsal root ganglion., ${ }^{5,6}$ The SSEP is recorded to clarify the functional continuity of the dorsal root sensory fibers with the spinal cord, ${ }^{4,8,9}$ but its clinical relevance rests largely on the assumption that the ventral (primarily motor) and dorsal (primarily sensory) nerve rootlets are never independently avulsed. False-positive SSEPs (when the ventral rootlet alone has been avulsed) may lead to futile attempts at nerve grafting, whereas false negatives (when the dorsal rootlet alone has been avulsed) may result in lost opportunities for grafting. Because partial root avulsions (intact ventral rootlets and avulsed dorsal rootlets or vice versa) have been reported in as many as $11 \%$ of patients with brachial plexus avulsions, ${ }^{1}$ the utility of NAP and SSEP studies may be limited in that neither method specifically assesses the functional integrity of motor nerve fibers from the anterior horn cell to peripheral nerves. Because motor recovery is the main objective of brachial plexus surgery, neurogenic MEPs may be useful in evaluating the functional continuity of motor nerve fibers with the spinal cord. 


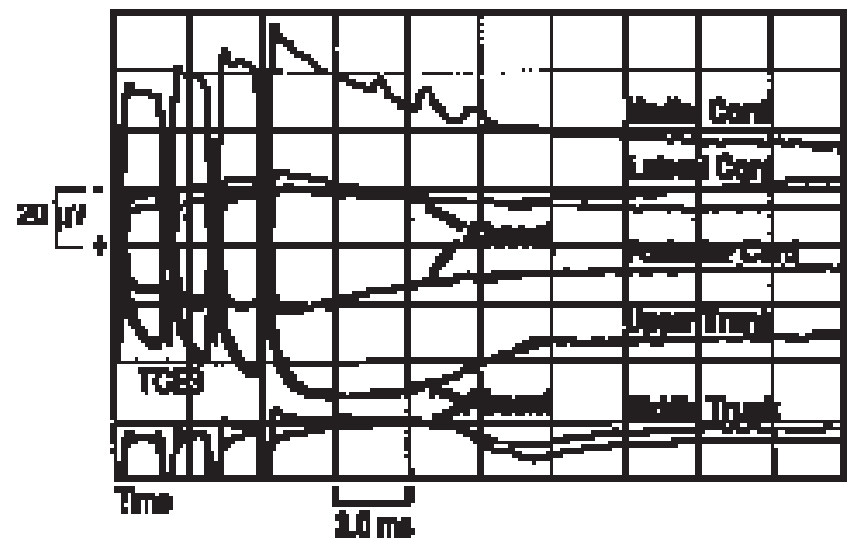

FIG. 1. Tracing showing the neurogenic MEPs recorded from different elements of the brachial plexus after TCES (C4 anode, 350 $\mathrm{V}$, train of four stimuli, interstimulus interval $2 \mathrm{msec}$ ). A neurogenic MEP was present in readings from the medial cord, but absent in all other elements of the brachial plexus.

\section{Case Report}

History and Examination. The patient was a 50-year-old man who fell to the ground from a height of approximately 30 feet. He suffered multiple spine fractures and sustained complete T-2 paraplegia from a fracture/dislocation. He also had multiple left rib fractures with flail chest, a right scapula fracture, and a left brachial plexus injury. Clinical examination of the left upper extremity demonstrated a complete upper plexus lesion (Erb palsy) with no clinical recovery after 4.5 months. On motor examination, he had complete absence of power in arm abduction, elbow flexion, elbow extension, forearm supination, wrist extension, and finger extension. The intraoperative use of TCES was approved by the Research Ethics Board at our institution, and informed consent was obtained from the patient before surgery.

Neuroimaging Studies. A preoperative computerized tomography myelogram yielded results consistent with probable C-5, C-6, and C-7 nerve root avulsions.

Anesthetic Agents. Induction of anesthesia was performed using a propofol and forane mixture. After induction, anesthesia was maintained with forane $(0.6 \%)$ and fentanyl.

Neurogenic MEPs. The TCES was performed with a stimulator (Digitimer D185; Digitimer Ltd., Hertfordshire, UK) that was triggered by the evoked potential machine. Stimulation was delivered through gold cup electrodes (AstroMed, Inc., West Warwick, RI) placed on the surface of the scalp at positions C3 and C4 (International 10-20 system). Multipulse TCES was completed with a train of four stimuli by using an interstimulus interval of $2 \mathrm{msec}$, a stimulus duration of $50 \mu \mathrm{sec}$, and the $\mathrm{C} 4$ location as the anode. The stimulus intensity was $100 \mathrm{~V}$ greater than that for the neurogenic MEP threshold. Neurogenic MEPs were directly recorded from selected sites of the exposed brachial plexus by using a bipolar hook electrode. The amplifier gain was 20,000, with a bandpass of 10 to $2000 \mathrm{~Hz}$ (Cadwell Laboratories, Kennewick, WA). Each neurogenic MEP recording was repeated and superimposed for waveform reproducibility.

Nerve Action Potentials. Selected portions of the exposed

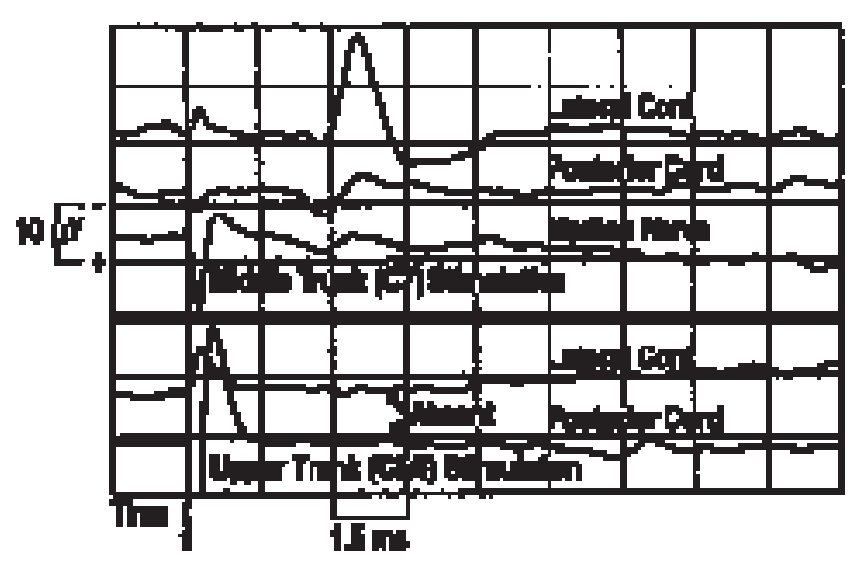

FIG. 2. Tracing showing that NAPs were present from the later$\mathrm{al}$ and posterior cords of the brachial plexus and the median nerve after direct stimulation of the middle trunk. In contrast, NAPs were absent from the lateral and posterior cord after stimulation of the upper trunk.

brachial plexus were directly stimulated with a tripolar hook electrode ${ }^{10}$ by using a monophasic rectangular pulse ranging from 3.3 to $6.7 \mathrm{~mA}$ with a 0.2 -msec duration. Stimulus intensity for each element of the brachial plexus was 1.5 times the stimulus intensity necessary to reach the NAP threshold in that element. The NAPs were recorded using the same bipolar hook electrode used in the neurogenic MEP recordings. The amplifier gain was 20,000 and the recording bandpass was 30 to $1000 \mathrm{~Hz}$. The NAP onset latency (the first negative deflection), and the NAP amplitude (NAP onset to first negative peak) were measured. The distance between the site of stimulation and the site of recording was measured and then divided by the NAP onset latency to calculate conduction velocity for each response.

Somatosensory Evoked Potentials. Selected elements of the exposed brachial plexus were directly stimulated by the same tripolar hook electrode used in the NAP study (6.7$\mathrm{mA}$ stimulus intensity, $0.2-\mathrm{msec}$ pulse width, and $3.13-\mathrm{Hz}$ stimulus rate). The SSEP recordings were obtained using platinum subdermal needle electrodes (Astro-Med, Inc.) placed on the scalp. The two recording electrode pairs for the SSEPs were as follows: 1) the C-2 spinous process (electrode $\mathrm{Cv} 2$ ) referenced to a midfrontal location at the hairline (electrode Fpz); and 2) the contralateral centroparietal scalp (electrode $\mathrm{C}^{\prime}$ ') referenced to Fpz. The amplifier gain was 50,000 and the bandpass was 10 to $500 \mathrm{~Hz}$ for the Cv2-Fpz (neck) electrode pair and 30 to $500 \mathrm{~Hz}$ for the C4'-Fpz (scalp) electrode pair. There were 66 to 195 responses included in each mean; the calculation of each mean was repeated to verify the reproducibility of the recordings. The latency of P/N13 (recorded from the neck), N20 (recorded from the scalp), and the amplitude of the N20-P25 waveform were measured.

\section{Results}

\section{Neurogenic MEPs}

Neurogenic MEPs containing three peaks were recorded as present in the medial cord of the brachial plexus after a train of four TCESs by using a stimulus intensity of $350 \mathrm{~V}$ 
(Fig. 1). During three trials, the onset latency to the first peak varied between 10.3 and $10.4 \mathrm{msec}$, the second peak was consistent at $12.2 \mathrm{msec}$, and the third peak varied between 14.1 and $14.3 \mathrm{msec}$. The first and second peaks were present in all three trials, but the third peak was present in two of three trials. The onset-to-peak amplitude for the first peak varied between 1.4 and $3.2 \mu \mathrm{V}$, the second peak between 4.6 and $7.9 \mu \mathrm{V}$, and the third peak was consistent at $4.6 \mu \mathrm{V}$ from trial to trial. In contrast, neurogenic MEPs were absent from the middle trunk, upper trunk, and the lateral and posterior cords after TCES.

\section{Nerve Action Potentials}

For NAPs assessed after stimulation of the middle trunk, with subsequent recordings from the lateral and posterior cords and the median nerve, responses were present, with conduction velocities of 44,42 , and $42 \mathrm{~m} / \mathrm{sec}$, respectively (Fig. 2). The onset-to-peak amplitude of the NAP was 18, 7 , and $3 \mu \mathrm{V}$, respectively. Responses were absent after stimulation of the upper trunk and subsequent recordings from the lateral and posterior cords.

\section{Somatosensory Evoked Potentials}

Somatosensory evoked potential recordings elicited from medial cord stimulation showed that subcortical and cortical responses were present (Fig. 3). The latency of the P/ $\mathrm{N} 13$ response was $8.8 \mathrm{msec}$ and the onset-to-peak amplitude was $1.9 \mu \mathrm{V}$. The onset latency of N20 was $16.1 \mathrm{msec}$ and the N20-P25 amplitude was $1.9 \mu \mathrm{V}$. Subcortical and cortical responses were absent after upper and middle trunk stimulation.

\section{Interpretation of Neurophysiological Results}

Recordings in which neurogenic MEPs and SSEPs were present provided evidence that the C-8 and T- 1 ventral and dorsal roots were functionally continuous with the spinal cord and that the related peripheral nerve was intact. The presence of NAPs, coupled with the absence of neurogenic MEPs and SSEPs on recordings, demonstrated that the peripheral nerve distal to the $\mathrm{C}-7$ dorsal root ganglion was intact, whereas the C-7 ventral and dorsal roots were avulsed from the spinal cord. Therefore, there was a $\mathrm{C}-7$ preganglionic lesion. Absence of NAPs showed that the peripheral nerve distal to the C-5 and C-6 dorsal root ganglions was disrupted, therefore indicating a C-5 and C-6 postganglionic lesion. Absence of neurogenic MEP and SSEP responses indicated C-5 and C-6 ventral and dorsal root avulsions. The possibility of a C-5 and C-6 preganglionic lesion remained undetermined by neurophysiological evaluation, however, because the absence of NAPs on the recordings precluded the ability to make such an assessment. It was possible that the neurogenic MEP recordings and SSEP stimulation were performed on disrupted nerve. Indeed, there was visible scarring of the C-5 and C- 6 spinal nerves that involved the upper trunk, the anterior and posterior divisions, as well as the suprascapular nerve. The gross appearance of these elements indicated that they were abnormal.

\section{Discussion}

The value of recording intraoperative SSEPs and NAPs

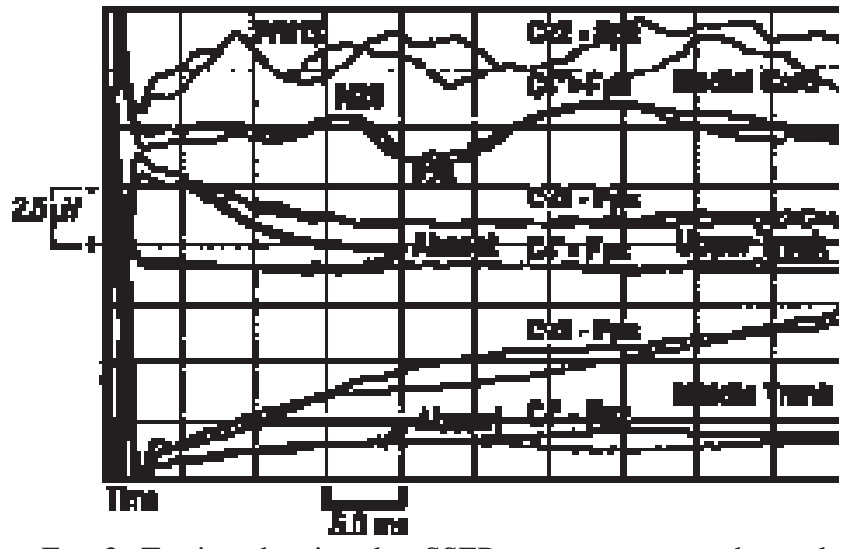

FIG. 3. Tracing showing that SSEPs were present at the neck (Cv2-Fpz) and scalp (C4'-Fpz) electrode pairs after direct stimulation of the medial cord. The SSEPs from the neck and scalp were absent after upper and middle trunk stimulation. Each trace is the mean of 66 to 195 responses. N20, P/N13, and P25 denote electrode positions.

during brachial plexus surgery has been widely acknowledged. Considering the limitations of NAPs and SSEPs, however, and because the primary purpose of surgical intervention is motor recovery, it is surprising that intraoperative neurogenic MEPs have received considerably less attention.

\section{Neurogenic MEPs}

Intraoperative neurogenic MEP studies were successfully performed to clarify specifically the functional integrity of motor fibers to the spinal cord at several cervical levels, as well as the status of motor fibers proximal to the brachial plexus exposure. Absence of neurogenic MEPs on recordings was used to avoid peripheral grafting from a cervical spinal nerve (in this case C-7) in which the proximal stump had degenerated, a situation in which motor recovery would not occur. If neurogenic MEPs were present in nerve roots originally believed to be avulsed, this information would have indicated an unexpected opportunity for peripheral nerve grafting to the proximal stump.

It has been shown that dorsal sacral nerve roots in humans contain some efferent fibers that are thought to belong to the same efferent fiber group as those in the ventral nerve roots, except that they grew through the wrong (dorsal) exit. $^{7}$ If these efferent fibers are also present in the dorsal cervical nerve roots, then a neurogenic MEP may be present even when the ventral root is avulsed. In that case, the presence of neurogenic MEPs would still indicate continuity of motor nerve fibers with motor neuron cell bodies (through the dorsal root). Further refinement of the neurogenic MEP modality may be necessary to allow discrimination of a discrete ventral nerve root avulsion.

\section{Somatosensory Evoked Potentials}

The neurogenic MEP and SSEP studies were complementary. The SSEPs permitted evaluation of the functional continuity of large-diameter sensory fibers (the dorsal root) to the spinal cord, whereas the neurogenic MEPs allowed for the evaluation of the functional continuity of mo- 
tor nerve fibers. There are reports of sensory fibers in ventral nerve roots, but these are limited to unmyelinated fibers that do not mediate SSEPs. ${ }^{2}$ Neurogenic MEP and SSEP studies used together provided a more complete analysis of the functional integrity of the nerve roots than the use of a single test.

\section{Nerve Action Potentials}

The importance of NAP findings was illustrated in this case study. The primary objective of NAPs was to assess mixed peripheral nerve function distal to the dorsal root ganglion, thus providing additional information to distinguish between pre- and postganglionic lesions. Nevertheless, we also found this method useful for clarifying the absence of SSEPs and neurogenic MEPs on recordings. For example, when the NAP was absent from the portion of the upper trunk selected for neurogenic MEP recording and SSEP stimulation, this confounded the assessment of the nerve roots because the neurogenic MEP and SSEP studies may have been performed on abnormal nerve. In contrast, when the NAP was present, as it was from the middle trunk, the absence of neurogenic MEP and SSEP findings could then be used to comment on the functional continuity of motor and sensory fibers to the spinal cord. The findings from all three electrophysiological methods were important for gaining an accurate picture of brachial plexus function in the operating room.

\section{Transcranial Electrical Stimulation}

The repetitive stimulation method that we used was a modification of the one described by Turkof, et al., ${ }^{11}$ who used a single TCES pulse of 750-V amplitude and 100- $\mu$ sec duration delivered through two silver-plate electrodes. The neurogenic MEPs that we recorded after repetitive TCES were obtained at considerably lower stimulus intensities $(350 \mathrm{~V})$ than those recorded after single-pulse TCES. This is because appropriately timed repetitive TCES (interstimulus interval $1.5-2 \mathrm{msec}$ ) is more effective in depolarizing alpha motor neurons in the presence of anesthesia than singlepulse TCES. ${ }^{3}$

\section{Conclusions}

For the first time, NAPs, SSEPs, and neurogenic MEPs were obtained from elements of the brachial plexus in the same patient. Although neurogenic MEPs and SSEPs should not replace the NAP for the purpose of intraoperative assessment of brachial plexus injuries, they may serve as a useful adjunct to NAPs in patients with proximal bra- chial plexus injuries. We believe that the combination of these techniques provides a more comprehensive assessment of intraoperative peripheral nerve function and promotes accurate localization of the lesion sites during brachial plexus surgery.

\section{References}

1. Carvalho GA, Nikkhah G, Matthies C, et al: Diagnosis of root avulsions in traumatic brachial plexus injuries: value of computerized tomography myelography and magnetic resonance imaging. J Neurosurg 86:69-76, 1997

2. Coggeshall RE, Applebaum ML, Fazen M, et al: Unmyelinated axons in human ventral roots, a possible explanation for the failure of dorsal rhizotomy to relieve pain. Brain 98:157-166, 1975

3. Houlden DA, Schwartz ML, Tator CH, et al: Spinal cord-evoked potentials and muscle responses evoked by transcranial magnetic stimulation in 10 awake human subjects. J Neurosci 19: 1855-1862, 1999

4. Jones SJ: Investigation of brachial plexus traction lesions by peripheral and spinal somatosensory evoked potentials. J Neurol Neurosurg Psychiatry 42:107-116, 1979

5. Kline DG, Happel LT: Penfield Lecture. A quarter century's experience with intraoperative nerve action potential recording. Can J Neurol Sci 20:3-10, 1993

6. Oberle J, Antoniadis G, Rath SA, et al: Value of nerve action potentials in the surgical management of traumatic nerve lesions. Neurosurgery 41:1337-1344, 1997

7. Schalow G: Ventral root afferent and dorsal root efferent fibres in dog and human lower sacral nerve roots. Gen Physiol Biophys 11:123-131, 1992

8. Sugioka H: Evoked potentials in the investigation of traumatic lesions of the peripheral nerve and the brachial plexus. Clin Orthop 184:85-92, 1984

9. Sugioka H, Tsuyama N, Hara T, et al: Investigation of brachial plexus injuries by intraoperative cortical somatosensory evoked potentials. Arch Orthop Trauma Surg 99:143-151, 1982

10. Tiel RL, Happel LT Jr, Kline DG: Nerve action potential recording method and equipment. Neurosurgery 39:103-109, 1996

11. Turkof E, Millesi H, Turkof R, et al: Intraoperative electroneurodiagnostics (transcranial electrical motor evoked potentials) to evaluate the functional status of anterior spinal roots and spinal nerves during brachial plexus surgery. Plast Reconstr Surg 99: 1632-1641, 1997

Manuscript received July 10, 2002.

Accepted in final form November 26, 2002.

Address reprint requests to: David Houlden, Ph.D., Suite B508, Sunnybrook and Women's College Health Sciences Centre, 2075 Bayview Avenue, Toronto, Ontario M4N 3M5, Canada. email: david.houlden@swchsc.on.ca. 